Article

\title{
Performance Analysis of Interference-Aware Multi-Packet Reception Networks
}

\author{
Antonio Furtado ${ }^{1, *,+(\mathbb{D})}$, Rodolfo Oliveira ${ }^{1,2, *,+(\mathbb{D})}$, Luis Bernardo ${ }^{1,2,+}+\mathbb{D}$ and Rui Dinis $1,2,+(\mathbb{D}$ \\ 1 Instituto de Telecomunicações, 1049-001 Lisboa, Portugal \\ 2 NOVA School of Science and Technology, Universidade Nova de Lisboa, 2829-516 Caparica, Portugal \\ * Correspondence: antonio.furtado@lx.it.pt (A.F.); rado@fct.unl.pt (R.O.) \\ + These authors contributed equally to this work.
}

Received: 28 February 2020; Accepted: 15 April 2020; Published: 20 April 2020

\begin{abstract}
Decentralized wireless networks are gaining increasing popularity as they do not need a fixed infrastructure. Simultaneously, multiple research initiatives have led to different findings at the PHY layer of the wireless communication systems, which include Multi-Packet Reception (MPR) techniques that enable a receiver to decode multiple packets that are transmitted simultaneously. However, the distributed nature of decentralized wireless networks demands different network control policies that should take into account the MPR capabilities to increase the network performance. This work studies the performance of a wireless network composed of multiple transmitters that are willing to transmit to a single receiver. This receiver has MPR capability and adopts an Energy-based Sensing (EBS) technique to enable uplink users' transmissions without interfering with the ongoing transmissions from other transmitters. The first remark to be made is that the MPR technique performance depends on the channel propagation conditions and on the amount of time the receiver needs to detect the spectrum's occupancy state. However, it is shown that by increasing the number of samples needed to increase the sensing accuracy, the receiver may degrade its throughput, namely if the receiver is equipped with a single radio, that is sequentially used for sensing and transmitting (split-phase operation). The results presented in the paper show the impact of the channel propagation condition and EBS parameterization on wireless network throughput and the cases where the receiver MPR capture performance is greatly improved by the use of a spectrum sensing technique.
\end{abstract}

Keywords: multi-packet reception; spectrum sensing; aggregate interference

\section{Introduction}

The exponential growth of wireless technologies and the increase of Internet of Things (IoT) and Machine-to-Machine (M2M) systems raise multiple challenges to the current wireless network systems [1]. Particularly in decentralized wireless networks, which include sensor networks and ad hoc vehicular wireless networks, the lack of wired infrastructure poses unique challenges in the design of the physical (PHY) and medium access control (MAC) layers. Although it is expected a huge increase in data demand and number of wireless devices, in the literature several works have demonstrated that decentralized wireless networks have scalability problems [2]. Focusing on overcoming theses scalability issues, multiple works including [3,4] have proposed the adoption of MPR techniques to increase the capacity of decentralized wireless networks. Different from Single-packet reception (SPR) techniques, which only allow one reception at a time, MPR PHY layer capability enables multiple packets to be decoded simultaneously at the receiver.

In the decentralized wireless networks, nodes simultaneously compete for the network resources. The competition of the nodes to access the medium is a central objective of the MAC protocols design. 
MAC protocols play an important role in terms of the throughput of decentralized wireless networks [5]. Additionally, several distributed protocols (such as IEEE 802.11 and IEEE 802.15.4) operate in shared Industrial Scientific Medical bands (e.g., 2.4 GHz). In such shared bands the interference level is usually high because they support different wireless technologies. One critical issue in decentralized wireless networks operating in shared bands is the problem of dealing with spatial interference, which arises from the need of sharing the same wireless channel controlled in a decentralized way. In a shared channel multiple interferers may decrease the communication performance because they are not controlled by a central entity capable of mitigating the spatial interference. This fact is of particular importance as the density of nodes increase, because the nodes far away from the receiver may also cause non-negligible interference.

The distributed nature and inherent flexibility features of the decentralized wireless networks have been responsible for the development of MAC protocols mainly based on Carrier-Sense Multiple Access (CSMA). The Carrier-Sense mechanism enables the sensing of the interference level before starting the communication process and thus undesired levels of interference in shared channels are avoided. Thereby the CSMA based protocols provide a significant performance improvement over the ALOHA based MAC protocols [3]. Multiple works have already analyzed the performance CSMA and ALOHA MAC protocols for decentralized wireless networks [6-10]. The main focus of these works was to define a threshold for the ratio between the sensing time and the transmitting time in which CSMA can outperform Aloha [6]. Some of these works assumed that the amount of time that is used to sense the channel is much lower than the amount of time used for data transmission. This assumption might be valid for the traditional decentralized wireless networks (e.g., IEEE 802.11). However, it can be unrealistic when considering IoT and M2M systems where the communication profile is typically characterized by data packets of short duration [11]. Considering this issue, in [7] and [8] the authors compared the performance of CSMA and ALOHA networks and concluded that for low Signal-to-Noise Ratio (SNR) and short data packets duration the ALOHA protocol achieved higher performance. In [8] the network performance of an SPR based PHY layer was characterized based on the capture model, which considered the propagation effects due to small-scale fading, the decision threshold that characterizes the receiving system, as well as the noise at the receiver.

Usually, CSMA schemes are adopted for single-capture reception where only a single transmitter is considered. In CSMA the main goal is to avoid collisions between the different nodes competing for the channel. To achieve this goal all CSMA transmitters first sense the channel to defer its transmission when the channel is found busy. By deferring its transmission they decrease the probability of accessing the channel, decreasing the probability of collision between two or more transmitters.

CSMA schemes have already been proposed for MPR PHY-based networks. In these works, the transmitters sense the channel before transmitting to regulate their medium access probability according to the channel activity. The performance of CSMA networks considering MPR capabilities has been studied in $[9,10,12,13]$ assuming a constant receiving capability where the optimal number of transmitters is greater than one. The work in [9] studies the scalability of MPR PHY schemes for wireless local area networks. In [10] the authors study cooperative contention schemes adopting CSMA medium access with MPR physical-layer techniques and evaluate the particular scenario when the MPR transmitters only sporadically have packets to transmit. The work in [14] also considers an MPR-based network, where the transmitters adopted a CSMA medium access operation. This work is particularly focused on characterizing the case when the multiple packets are asynchronously arriving at the receiver, which is of particular importance for the backoff mechanism also considered in the paper. $\tau$-persistent MPR CSMA networks have been studied in [12]. MPR CSMA networks operating according to the IEEE 802.11 MAC scheme were considered in [13] and the performance of conjoining CSMA was investigated. Several non-CSMA MPR schemes were also proposed. A coded slotted ALOHA MAC with MPR capability was presented in [15]. Random access non-CSMA MPR operation has been considered in [16], where the authors assume simultaneous multipacket channel estimation 
and reception over random channels. In [17] an adaptive grant-free access scheme with multi-packet reception was evaluated.

Differently from the previous works, the focus of our work is not on avoiding collisions but rather to determine when should the MPR transmitters start transmitting to maximize the throughput at the receiver. Consequently, the channel sensing is not performed by the transmitters but by the receiver. The receiver can thus indicate when the various transmitters should initiate the transmission, depending on the level of interference sensed at the receiver. We compare the advantage of adopting a sensing scheme at the receiver, by studying if the sensing can increase the MPR throughput. The performance of the channel sensing mechanism is evaluated in a first step, and the throughput of a wireless network with MPR capabilities is then analyzed to identify the advantages of performing channel sensing. We consider a wireless network composed of multiple users that transmit to a single receiver. Considering that the wireless network operates in a shared band scenario, the receiver senses the channel to avoid undesirable interference caused by other communications that are occurring in the sensed channel. When the level of interference is high the receiver and the transmitters may postpone the communication until lower interference levels are found in the channel. By doing so, the receiver node can increase the probability of successfully receiving the packet(s). Simultaneously, the transmitters also guarantee a certain level of protection to other communications (primary communications) that are already using the channel in the vicinity of the receiver.

The main contributions of this paper include:

- The assumption of an innovative operation mode, where the transmission of the MPR nodes depends on the level of interference sensed by the receiver;

- The derivation of the sensing threshold to parameterize the carrier sense scheme at the receiver, which is used by the receiver to signal that the multiple MPR nodes can initiate the transmission. The sensing threshold takes into account the spatial distribution of the nodes, fading conditions and a circular spatial sensing region centered at the receiver;

- The characterization of the uplink throughput achieved when no interferers are sensed in the carrier sensing region of the receiver and multiple MPR nodes simultaneously transmit;

- A detailed study of the upper bound of the conditional throughput achieved by the wireless network that takes into account the carrier sense duration and the interference of other ongoing communications located outside the carrier sensing region.

In the next section we describe the considered system. Section 3 describes the steps involved to characterize the aggregate power and envelope signal received by the sensing node from the transmitters located in a circular ring. In Section 4 the receiver's performance of the EBS technique and the capture capability are characterized, which are also validated through simulation. Finally, Section 5 characterizes the spectrum sensing performance when multiple transmitters are considered and evaluates its impact on the throughput. Final conclusions are drawn in Section 6.

Notations: In this work, $f_{X}(),. M_{X}($.$) and \varphi_{X}($.$) represent the Probability Density Function (PDF),$ the Moment-Generating Function (MGF) and the Characteristic Function (CF) of a Random Variable (RV) $X$, respectively. $P[X=x]$ and $E[X]$ represent the probability and the expectation of the RV $X$, respectively. $\Gamma(x)$ and $\Gamma(s, x)$ represent the Gamma function and the upper incomplete Gamma function, respectively. $\mathcal{Q}(x)$ and ${ }_{2} \mathrm{~F}_{1}$ represent the complementary distribution function of the standard Normal and the Gauss Hypergeometric function, respectively.

\section{System Description}

This work considers the coexistence of two wireless networks as illustrated in Figure 1, which share the same band. One of the wireless networks is composed of Primary Users (PUs) (i.e., primary network), which act as interferers to the communications of the Secundary Users (SUs). The other wireless network is seen as the network of interest formed by SUs, which opportunistically access the channel in the absence of PUs. This wireless network is named secondary network. 
Regarding the secondary network, we consider that $n^{\mathrm{SU}}$ nodes take advantage of the MPR-based PHY layer to transmit data simultaneously to a single $\mathrm{SU}$ (the receiver $\mathrm{SU}_{\mathrm{rx}}$ ), as represented in Figure 1.

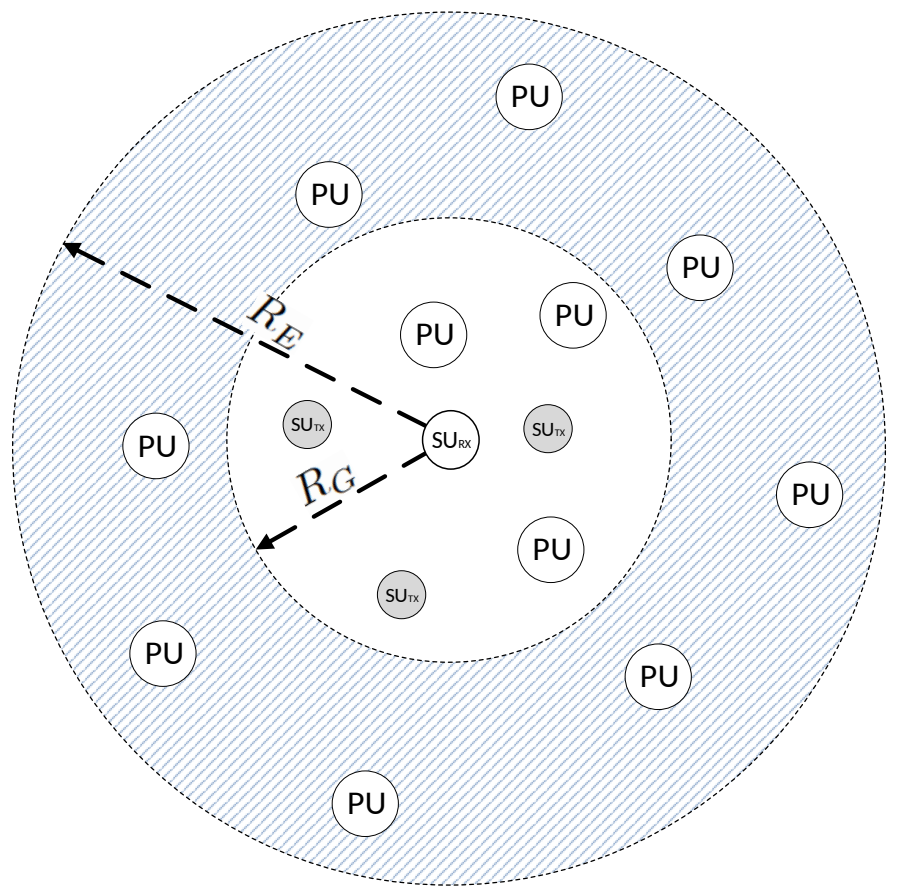

Figure 1. Sensing (white) and interference (gray) regions.

The node $\mathrm{SU}_{\mathrm{rx}}$ has a given carrier sensing range, which is limited by the radius $R_{G}$. Within the carrier sensing region (white zone in the figure, with correspondent area of $A_{\text {in }}=\pi R_{G}{ }^{2}$ ), an active PU must be detected with a given probability $P_{D}$ to guarantee that the receiver node $\mathrm{SU}_{\mathrm{rx}}$ avoids communicating when the interference level is high. Simultaneously, the SU transmitters guarantee a certain level of protection to the PUs. PUs can also be located outside the carrier sensing region (gray zone), causing interference to the SUs simultaneously transmitting to the SU's receiver.

Each PU and SU is equipped with an omni-directional antenna and the SUs and PUs share the same frequency band. Time is divided into equal size slots, which are grouped into frames with a duration of $T_{F}$.

Single radio SUs are considered, meaning that SUs are equipped with a single transceiver. Therefore, the SUs are unable to sense and transmit simultaneously. Due to this limitation, SUs adopt an operation cycle where sensing and transmission operations occur in a consecutive manner. $\mathrm{SU}_{\mathrm{rx}}$ starts to sense the spectrum during a fixed amount of time (sensing period). If the $\mathrm{SU}_{\mathrm{rx}}$ senses the channel as idle then the $\mathrm{SU}_{\mathrm{rx}}$ allows the $\mathrm{SUs}$ to jointly transmit in the sensed band during a fixed amount of time (transmission period). SUs repeat the operation cycle periodically to minimize the amount of interference caused to PU and mitigate the interference caused to SUs' transmissions. In this way, each SU may access the channel opportunistically, when one or more PU do not use the channel, as considered in [18]. $\mathrm{SU}_{\mathrm{rx}}$ adopts an EBS technique. SU's sensing and transmitting period durations are denoted by $T_{S}$ and $T_{D}$, respectively. In this work, we assume that all SUs are synchronized.

The time frame of the SUs is divided into $N_{T}$ slots where each slot duration is given by the channel sampling period adopted by the energy detector. The first $N_{S}$ slots are allocated to the spectrum sensing task (for channel sampling) and the remaining ones $\left(N_{S}+1\right.$ to $\left.N_{T}\right)$ are used to access the channel (for transmission, whenever possible). 
The PUs are distributed within a certain area $A^{\mathrm{PU}}=\pi\left(R_{E}\right)^{2}$ encircling the SU receiver. The number of active PUs is represented by a RV $N^{\mathrm{PU}}$. In this work, it is considered that the number of active PUs is distributed according to a 2D Poisson point process, with distribution,

$$
\mathrm{P}\left[N^{\mathrm{PU}}=n\right]=\frac{\left(\rho_{\mathrm{ON}} \tau^{\mathrm{PU}} A_{l}\right)^{n}}{n !} e^{-\rho_{\mathrm{ON}} \tau^{\mathrm{PU}} A_{l}}, n=0,1, \ldots,
$$

where $\tau^{\mathrm{PU}}$ represents the PU's spatial density, $\rho_{\mathrm{ON}}$ is the probability of finding a PU active and $A_{l}$ represents the area where the PUs are distributed (e.g., for the total area where the PUs are distributed $A_{l}=A^{\mathrm{PU}}$ ).

We assume that all the nodes of both networks are uniformly distributed within an annulus with area $A_{l}=\pi\left(\left(R_{o}\right)^{2}-\left(R_{i}\right)^{2}\right)$ encircling the receiver, the PDF of the distance between the $k$-th transmitter and the receiver, $R_{k}$, can be written as the ratio between the perimeter of the circle with radius $r$ and the total area $A_{l}$, as follows

$$
f_{R_{k}}(r)=\left\{\begin{array}{cc}
\frac{2 \pi r}{A_{l}} & R_{i}<r_{i}<R_{o} \\
0, & \text { otherwise }
\end{array}\right.
$$

$R_{i}$ and $R_{o}$ represent the inner radius and outer radius of the region where the transmitters are located, respectively. For the SUs network and the PUs inside the carrier sensing region, $R_{i}$ and $R_{o}$ are equal to zero and $R_{G}$, respectively. For the PUs that are located outside of the carrier sensing region, $R_{i}$ and $R_{o}$ are equal to $R_{G}$ and $R_{E}$, respectively.

The small-scale and large-scale fading are considered as being distributed according to a Rayleigh distribution and Lognormal distribution, respectively [19]. The Rayleigh fading is defined by the mean $2 \sigma_{\zeta}^{2}$. In this work we adopt a normalized small-scale mean power (i.e., $2 \sigma_{\zeta}^{2}=1$ ). The Lognormal fading is parameterized by the scale parameter $\sigma_{\xi}$ and location parameter $\mu_{\xi}$, and in what follows we assumed an average unitary gain (i.e., $\mu_{\xi}=-\sigma_{\xi}^{2} / 2$ ). As proposed in [20], the composite effects of small-scale and large-scale fading is assumed to be approximated by the following Gamma distribution

$$
f_{\Psi_{k}}(\psi)=\frac{\psi^{\left(k_{\xi}-1\right)}}{\Gamma(k) \theta_{\xi} k_{\xi}} e^{-\frac{\psi}{\theta_{\tilde{\xi}}}},
$$

where $\theta_{\xi}$ and $k_{\xi}$ are given by $\left(2 e^{\sigma_{\tilde{\xi}}^{2}}-1\right)$ and $1 / \theta_{\xi}$, respectively.

\section{Amplitude and Power of the Aggregate Interference}

This section presents the characterization of the aggregate powers and aggregate envelope signals observed at the receiver $\mathrm{SU}_{\mathrm{rx}}$. The characterization is derived considering both SUs' and PUs' networks, the noise at the receiver, and for two distinct scenarios of channel propagation conditions (i.e., a scenario with both path loss and fading effects, and another scenario considering only the path loss effect).

Taking into account the $\mathrm{SU}$ s operation cycle, we highlight that the $\mathrm{SU}_{\mathrm{rx}}$ starts to sense the spectrum during a fixed amount of time $T_{S}$ and then if the channel is sensed vacant the $n^{\mathrm{SU}}$ transmitters are allowed to transmit during a fixed amount of time $T_{D}$. It is considered that the receiver $\mathrm{SU}_{\mathrm{rx}}$ decides if the transmitters can proceed or postpone their transmission according to the spectrum sensing outcome. Moreover, we assume that during the transmission period $T_{D}$ no PU's transmission occurs within the sensing region when $\mathrm{SUs}$ transmitters communicate with the receiver $\mathrm{SU}_{\mathrm{rx}}$. This assumption represents the best performance scenario, where the SUs' transmissions do not suffer from interference caused by the PUs located within the sensing region. 
Considering that the channel is correctly sensed vacant, the total aggregate power received at the $\mathrm{SU}_{\mathrm{rx}}$ from the multiple transmissions, is given by

$$
\Lambda^{\mathrm{SU}}=N_{0}+\Xi^{\mathrm{SU}}+\Xi_{\text {out }}^{\mathrm{PU}}
$$

where $\Xi_{\text {out }}^{\mathrm{PU}}$ is the RV representing the aggregate power received from $n^{\mathrm{PU}}$ transmissions that are located outside of the transmission range. $\Xi^{\mathrm{SU}}$ is the $\mathrm{RV}$ representing the aggregate power received from the $n^{\mathrm{SU}} \mathrm{SU}$ transmissions. $N_{0}$ is a RV representing the noise power at the receiver, a zero-mean Additive White Gaussian Noise (AWGN) with variance $\sigma_{N_{0}}$ is assumed.

Let us consider a general network in which $n$ nodes located within the area $A=\pi\left(\left(R_{0}\right)^{2}-\left(R_{i}\right)^{2}\right)$ are transmitting. The aggregate power received at the receiver is given by

$$
\Xi=\sum_{k=1}^{n} P_{k}
$$

where $P_{k}$ is a RV representing the power received from the $k$-th transmitter located within $A$. Note that in the case of the SUs' network $n$ corresponds to the number of SUs simultaneously transmitting (i.e., $n^{\mathrm{SU}}$ ) which is assumed to be deterministic and known. For the case of the PUs' network, the number of PUs transmitting is assumed to be a RV distributed according to (1).

The power received by the node $\mathrm{SU}_{\mathrm{rx}}$ from a $\mathrm{SU}_{\mathrm{tx}}$ or a $\mathrm{PU}_{\mathrm{tx}}$ is given by

$$
P_{k}=P_{T} \Psi_{k}\left(R_{k}\right)^{-\alpha},
$$

where $P_{T}$ is a constant representing the transmitted power adopted by each network's node, and no power control is applied. The transmitted power $P_{T}$ is equal to $P_{T}^{\mathrm{SU}}$ and $P_{T}^{\mathrm{PU}}$ for the case of the SUs' network and PUs' network, respectively. The RV $R_{k}$ represents the distance between the $k$-th transmitter and the receiver, and $\alpha$ is the path loss coefficient. $\Psi_{k}$ is a RV that represents the fading observed in the channel between the receiver and the $k$-th transmitter.

Let $M_{P_{k}}$ represent the Moment-Generating Function (MGF) of the power received from the $k$-th transmitter. $M_{P_{k}}$ is defined as follows

$$
M_{P_{k}}(s)=\mathrm{E}_{P_{k}}\left[e^{s P_{k}}\right] .
$$

Using (2), (3) and (6), (7) is rewritten as follows

$$
M_{P_{k}}(s)=\mathrm{E}_{\Psi_{k}}\left[\mathrm{E}_{R_{k}}\left[e^{s P_{T} \Psi_{k}\left(R_{k}\right)^{-\alpha}}\right]\right]=\int_{-\infty}^{\infty} \int_{R_{i}}^{R_{o}} e^{s P_{T} \psi(r)^{-\alpha}} f_{R_{k}}(r) f_{\Psi_{k}}(\psi) \mathrm{d} r \mathrm{~d} \psi .
$$

In [21] the authors have derived the integral in (8). Using the MGF derived in the integral in ([21], Equation (20)), (8) is written as

$$
M_{P_{k}}(s)=\frac{2}{\left(R_{o}{ }^{2}-R_{i}{ }^{2}\right)\left(2+\alpha k_{\xi}\right)\left(s P_{T} \theta_{\xi}\right)^{k_{\xi}}} \cdot\left[R_{o}\left(2+\alpha k_{\xi}\right) \mathbb{I}\left(\frac{R_{o}^{\alpha}}{s}\right)-R_{i}\left(2+\alpha k_{\xi}\right) \mathbb{I}\left(\frac{R_{i}^{\alpha}}{s}\right)\right] .
$$

where $\mathbb{I}(z)={ }_{2} \mathrm{~F}_{1}\left(k_{\psi}, k_{\psi}+\frac{2}{\alpha}, 1+k_{\psi}+\frac{2}{\alpha},-\frac{z}{P_{T} \theta_{\psi}}\right)$.

Knowing that for a RV $X$ the $C F$ can be obtained from its MGF, i.e., $\varphi_{X}(t)=M_{X}(-i t)$, by using the MGF (9) the CF of $P_{k}, \varphi_{P_{k}}$, can be written as follows

$$
\varphi_{P_{k}}(t)=\frac{2}{\left(R_{o}^{2}-R_{i}^{2}\right)\left(2+\alpha k_{\xi}\right)\left(-i t P_{T} \theta_{\xi}\right)^{k_{\xi}}} \cdot\left[R_{o}\left(2+\alpha k_{\tilde{\xi}}\right) \mathbb{I}\left(\frac{i R_{o}{ }^{\alpha}}{t}\right)-R_{i}\left(2+\alpha k_{\tilde{\xi}}\right) \mathbb{I}\left(\frac{i R_{i}^{\alpha}}{t}\right)\right] .
$$


For the particular case where only path loss is considered as channel propagation effects, (7) is rewritten as $\mathrm{E}_{R_{k}}\left[e^{s P_{T}\left(R_{k}\right)^{-\alpha}}\right]$ (i.e., $\Psi_{k}=1$ in (6)) and the MGF and CF of $P_{k}$ are rewritten as follows

$$
\begin{aligned}
M_{P_{k}^{P L}}(s) & =\frac{2\left(-P_{T} s\right)^{2 / \alpha}}{\left(R_{o}^{2}-R_{i}^{2}\right) \alpha}\left(\Gamma\left[-\frac{2}{\alpha},-\frac{P_{T}}{R_{o}^{\alpha}} s\right]-\Gamma\left[-\frac{2}{\alpha},-\frac{P_{T}}{R_{i}^{\alpha}} s\right]\right), \\
\varphi_{P_{k}^{P L}}(t) & =\frac{2\left(i t P_{T}\right)^{2 / \alpha}}{\left(R_{o}{ }^{2}-R_{i}^{2}\right) \alpha}\left(\Gamma\left[-\frac{2}{\alpha}, \frac{P_{T}}{R_{o}^{\alpha}} i t\right]-\Gamma\left[-\frac{2}{\alpha}, \frac{P_{T}}{R_{i}^{\alpha}} i t\right]\right),
\end{aligned}
$$

respectively.

\subsection{Power Signals of Secondary Network}

Regarding the SUs' network, the number of nodes willing to transmit to the $\mathrm{SU}_{\mathrm{rx}}$ is known and equal to $n^{\mathrm{SU}}$. The CF of the aggregate power when $n^{\mathrm{SU}}$ nodes transmit is written as follows

$$
\varphi_{\Xi} \mathrm{SU}(t)=\varphi_{P_{1}}(t) \times \varphi_{P_{2}}(t) \times \cdots \times \varphi_{P_{n} \mathrm{SU}}(t) .
$$

The powers of the SUs signal powers are assumed to be i.i.d. RVs, $\varphi_{\Xi^{S U}}$ is rewritten as

$$
\varphi_{\Xi \mathrm{SU}}(t)=\prod_{k=1}^{n^{\mathrm{SU}}} \varphi_{P_{k}}(t),
$$

where $\varphi_{P_{k}}(t)$ is given by (10) when both path loss and fading effects are considered, or by (12) when only path loss effects are considered.

\subsection{Power and Envelope Signals of Primary Network}

In [21] the aggregate power of nodes that are distributed according to a 2D Poisson point process and located within an annulus is approximated through a Gamma distribution. Likewise, the interference caused by multiple PU nodes located within an area $A_{l}$ can be approximated by a Gamma distribution with PDF and CF given by,

$$
f_{\Xi \mathrm{PU}}(x) \approx \frac{x^{\left(k_{\mathrm{PU}}-1\right)}}{\Gamma\left(k_{\mathrm{PU}}\right) \theta_{\mathrm{PU}} k_{\mathrm{PU}}} e^{-\frac{x}{\theta_{\mathrm{PU}}}}
$$

and,

$$
\varphi_{\Xi^{\mathrm{PU}}}(t) \approx\left(1-i t \theta_{\mathrm{PU}}\right)^{-k_{\mathrm{PU}}}
$$

where the parameters $k_{\mathrm{PU}}$ and $\theta_{\mathrm{PU}}$ of the Gamma distribution are obtained by employing a moment matching method [20]. As in [21], the shape and the scale parameters of the Gamma distribution that characterizes the aggregate interference caused by the transmitters located within an area $A_{l}$ are respectively given by

$$
\begin{gathered}
k_{\mathrm{PU}}=\frac{2 \pi \rho_{O N} \tau^{\mathrm{PU}} P_{T}^{\mathrm{PU}} e^{\mu_{\xi}} \sqrt{e^{\sigma_{\xi}^{2}}}\left(R_{o}{ }^{2-\alpha}-R_{i}{ }^{2-\alpha}\right)}{2-\alpha}, \\
\theta_{\mathrm{PU}}=\frac{\pi \rho_{O N} \tau^{\mathrm{PU}}\left(\theta_{\xi} P_{T}^{\mathrm{PU}}\right)^{2} k_{\xi}\left(1+k_{\xi}\right)\left(R_{o}{ }^{2-2 \alpha}-R_{i}{ }^{2-2 \alpha}\right)}{1-\alpha} .
\end{gathered}
$$


In the particular case where only path loss is considered the parameters $k_{\mathrm{PU}}$ and $\theta_{\mathrm{PU}}$ are rewritten as

$$
\begin{gathered}
k_{\mathrm{PU}}=\frac{4 \pi \rho_{\mathrm{ON}} \tau^{\mathrm{PU}} P_{T}^{\mathrm{PU}}(\alpha-1)\left(R_{o}{ }^{\alpha} R_{i}{ }^{2}-R_{o}{ }^{2} R_{i}{ }^{\alpha}\right)^{2}}{(\alpha-2)^{2}\left(R_{o}{ }^{2 \alpha} R_{i}{ }^{2}-{R_{o}}^{2} R_{i}{ }^{2 \alpha}\right)}, \\
\theta_{\mathrm{PU}}=\frac{P_{T}^{\mathrm{PU}}(2-\alpha)\left(R_{o}{ }^{2-2 \alpha}-R_{i}{ }^{2-2 \alpha}\right)}{2(1-\alpha)\left({R_{o}}^{2-\alpha}-{R_{i}}^{2-\alpha}\right)} .
\end{gathered}
$$

The Gamma distribution represents the aggregate interference power received by $\mathrm{SU}_{\mathrm{rx}}$ from the PUs located within the area $A_{l}$. The envelope signal (amplitude) of the aggregate interference, $s_{\mathrm{PU}}$, is given by the square root of a Gamma distributed RV, which in [18] is approximated (the amplitude of the aggregate interference can be approximated by a Normal distribution when the number of PUs is enough to observe the Central Limit Theorem (CLT) conditions and the considered $R_{i}$ value is according to the far-field scenario) by a Normal distribution. Considering the Normal approximation proposed in [18], the resultant Normal distribution of the aggregate interference envelope has the following mean $\left(\mu_{\Xi^{\mathrm{PU}}}\right)$ and variance $\left(\sigma_{\Xi^{\mathrm{PU}}}^{2}\right)$

$$
\begin{gathered}
\mu_{\Xi \mathrm{PU}}=\sqrt{\theta_{\mathrm{PU}}} \frac{\Gamma\left(k_{\mathrm{PU}}+1 / 2\right)}{\Gamma\left(k_{\mathrm{PU}}\right)}, \\
\sigma_{\Xi}^{2}{ }_{\mathrm{PU}}=\theta_{\mathrm{PU}}\left(\frac{\Gamma\left(k_{\mathrm{PU}}+1\right)}{\Gamma\left(k_{\mathrm{PU}}\right)}-\frac{\Gamma\left(k_{\mathrm{PU}}+1 / 2\right)^{2}}{\Gamma\left(k_{\mathrm{PU}}\right)^{2}}\right) .
\end{gathered}
$$

\subsection{Noise Power Characterization}

Finally, the noise at the receiver, $N_{0}$, a Zero-mean AWGN is assumed. Since the AWGN follows a complex Normal distribution, $\mathcal{C N}\left(0, \sigma_{N_{0}}^{2}\right)$, its power is exponentially distributed and the respective $\mathrm{CF}$ is given by

$$
\varphi_{N_{0}}(t)=\frac{\sigma_{N_{0}}^{2}}{\sigma_{N_{0}}^{2}+i t} .
$$

\section{MPR-based PHY-layer Performance Characterization}

In this section we characterize the performance of the SU's MPR-based PHY layer and the throughput of the considered wireless network. The PHY layer with MPR capabilities of the SUs is characterized by the individual probability of successful reception and the average number of received packets when $n^{\mathrm{SU}}$ simultaneous transmissions occur.

\subsection{Spectrum Sensing Performance Characterization}

The aggregate interference approximation model presented in the Section 3.2 is now used to characterize performance of the EBS technique through the probabilities of detection and false alarm. Considering that the interference envelope caused by several PUs located inside and outside of sensing region can be approximated by Gaussian distributions, the PUs' activity detection within the sensing region can be employed by traditional binary hypothesis testing. In several works (e.g., [22-25]), the hypothesis testing was used by considering the hypotheses of only observing noise or a signal plus noise to indicate a vacant channel or an occupied channel, respectively. However, different hypotheses were presented in [18] to consider the presence of communication outside the sensing region. As in [18], we considered that a channel is considered vacant when only noise and a given amount of interference generated by PUs located outside the sensing region is observed; or a channel is declared occupied when in addition to noise and to the interference generated outside the sensing region, the PUs within the sensing region become active. 
To distinguish between vacant and occupied spectrum bands, the $S U_{\mathrm{rx}}$ samples the channel during the sensing period $T_{S}$. In each sample $n$ the two hypotheses can be distinguished

$$
\begin{array}{ll}
\mathcal{H}_{0}: r(n)=w(n)+s_{\text {out }}^{\mathrm{PU}}(n) & n=1,2, \ldots, N_{S} \\
\mathcal{H}_{1}: r(n)=w(n)+s_{\text {out }}^{\mathrm{PU}}(n)+s_{\text {in }}^{\mathrm{PU}}(n) & n=1,2, \ldots, N_{S},
\end{array}
$$

where $r(n)$ is the received signal by the $\mathrm{SU}_{\mathrm{rx}}$. The first condition, $\mathcal{H}_{0}$, represents the hypothesis corresponding to the absence of PUs inside the sensing region, while the second condition, $\mathcal{H}_{1}$, indicates the occurrence of PUs' activity within the sensing region. $s_{\text {out }}^{\mathrm{PU}}(n)$ and $s_{i n}^{\mathrm{PU}}(n)$ denote respectively the aggregate interference caused by PUs located outside and within the sensing region, i.e., the aggregate interference envelope generated by the PUs located within the areas $A_{\text {out }}=\pi\left(R_{E}^{2}-R_{G}^{2}\right)$ and $A_{\text {in }}=$ $\pi R_{G}^{2}$, respectively. From (19), $s_{\text {out }}(n)$ and $s_{i n}(n)$ may be approximated by a Normal random variable with mean and variance respectively denoted by $\mu_{i n}, \sigma_{i n}^{2}$ and $\mu_{\text {out }}, \sigma_{\text {out }}^{2}$. A zero-mean AWGN with $\sigma_{N_{0}}^{2}$ variance is assumed in this work (i.e., $w(n) \sim \mathcal{N}\left(0, \sigma_{N_{0}}^{2}\right)$ ).

Considering the work in [18], the performance of the EBS technique is characterized by the probabilities of detection $\left(P_{D}\right)$ and false alarm $\left(P_{F A}\right)$. The probability of false alarm represents the probability of receiver $\mathrm{SU}_{\mathrm{rx}}$ erroneously detect the present $\mathrm{PUs}$ within the sensing region. On other hand the probability of detection represents the probability of detecting correctly the PUs transmission within the sensing region. The probabilities of detection $\left(P_{D}\right)$ and false alarm $\left(P_{F A}\right)$ are represented by

$$
P_{F A}=\mathcal{Q}\left(\frac{\gamma-\left(N_{S}+\lambda_{\mathcal{H}_{0}}\right)\left(\sigma_{o u t}^{2}+\sigma_{N_{0}}^{2}\right)}{\sqrt{\left(2 N_{S}+4 \lambda_{\mathcal{H}_{0}}\right)\left(\sigma_{\text {out }}^{2}+\sigma_{N_{0}}^{2}\right)^{2}}}\right)
$$

and

$$
P_{D}=\mathcal{Q}\left(\frac{\gamma-\left(N_{S}+\lambda_{\mathcal{H}_{1}}\right)\left(\sigma_{o u t}^{2}+\sigma_{\text {in }}^{2}+\sigma_{N_{0}}^{2}\right)}{\sqrt{\left(2 N_{S}+4 \lambda_{\mathcal{H}_{1}}\right)\left(\sigma_{o u t}^{2}+\sigma_{\text {in }}^{2}+\sigma_{N_{0}}^{2}\right)^{2}}}\right)
$$

where $\lambda_{\mathcal{H}_{x}}$ are respectively given by

$$
\lambda_{\mathcal{H}_{x}}= \begin{cases}\lambda_{\mathcal{H}_{0}}=N_{S} \frac{\mu_{\text {out }}^{2}}{\sigma_{\text {out }}^{2}+\sigma_{N_{0}}^{2}}, & \mathcal{H}_{0}, \\ \lambda_{\mathcal{H}_{1}}=N_{S} \frac{\left(\mu_{\text {out }}+\mu_{\text {in }}\right)^{2}}{\sigma_{\text {in }}^{2}+\sigma_{\text {out }}^{2}+\sigma_{N_{0}}^{2}}, & \mathcal{H}_{1} .\end{cases}
$$

From (22) and (23) we observe that $P_{F A}$ and $P_{D}$ depend on the number of samples $\left(N_{S}\right)$, the energy threshold $(\gamma)$, the mean and the variance of the aggregate interference caused by the PUs located inside and outside of the sensing region.

The energy threshold which guarantees a level of protection to the PUs located within the sensing region and consequently avoids SUs transmissions to occur, is parameterized according to a given probability of detection. Given $P_{D}$, the energy threshold is parameterized to be the middle point between the averages of received energy in hypotheses $\mathcal{H}_{0}$ and $\mathcal{H}_{1}$. Based on these two conditions, 
the following criterion is used to define the minimum number of samples $\left(N_{S}\right)$ that guarantees a required level of protection to the PUs,

$$
\begin{array}{ll}
N_{S}^{*}= & \min N_{S} \\
\text { s.t. } & \\
& P_{D}=\chi, \\
& N_{S}>2 W T_{S}, \\
& \eta=\frac{\left(N_{S}+\lambda_{\mathcal{H}_{1}}\right)\left(\sigma_{\text {out }}^{2}+\sigma_{\text {in }}^{2}+\sigma_{N_{0}}^{2}\right)+\left(N_{S}+\lambda_{\mathcal{H}_{0}}\right)\left(\sigma_{\text {out }}^{2}+\sigma_{N_{0}}^{2}\right)}{2},
\end{array}
$$

where $N_{S}^{*}$ is the minimum number of samples to guarantee the expected level of protection $\chi$. $W$ represents the bandwidth of the sensed band. The Nyquist sampling rate is imposed by the constraint $N_{S}>2 W T_{S}$.

\subsection{Capture Performance Characterization}

In this subsection, we characterize the performance of the SU's MPR-based PHY-layer and the throughput of the SUs' network. The PHY layer with MPR capabilities of the SU is characterized by the individual probability of successful reception and the average number of received packets when $n^{\mathrm{SU}}$ simultaneous transmissions occur.

Following the capture condition defined in [26] the capture capability of decoding the signal received from transmitter $j$ is derived when the Signal-to-Interference-plus-Noise Ratio (SINR) of the $j$-th signal at the receiver is higher than a certain threshold $b$. Based on that, the necessary condition for successful reception of the signal from node $j$ is given as

$$
\frac{P_{j}^{\mathrm{SU}}}{\Lambda^{\mathrm{SU}}-P_{j}^{\mathrm{SU}}}>b .
$$

From (26), the probability of successful reception may be written as follows

$$
\mathrm{P}_{S}=\mathrm{P}\left[\frac{P_{j}^{\mathrm{SU}}}{\Lambda^{\mathrm{SU}}-P_{j}^{\mathrm{SU}}}>b\right]=1-\mathrm{P}\left[P_{j}^{\mathrm{SU}}-b \Lambda^{\mathrm{SU}}-b P_{j}^{\mathrm{SU}} \leq 0\right] .
$$

Let's consider a RV $\beta$ which is defined by $P_{j}^{\mathrm{SU}}-b \Lambda^{\mathrm{SU}}-b P_{j}^{\mathrm{SU}}$. From (27) and using $\beta$, the probability of successful packet reception can be written as

$$
\mathrm{P}_{S}=1-\mathrm{P}[\beta \leq 0]
$$

From (4), the RV $\beta$ is given by

$$
\beta=P_{j}^{\mathrm{SU}}-b \Xi^{\mathrm{SU}}-b \Xi_{\text {out }}^{\mathrm{PU}}-b N_{0}-b P_{j}^{\mathrm{SU}} .
$$

Considering that $P_{j}^{\mathrm{SU}}, \Xi^{\mathrm{SU}}, \Xi_{\text {out }}^{\mathrm{PU}}$ and $N_{0}$ are independent RVs, the CF of the RV $\beta$ is written as

$$
\begin{aligned}
\varphi_{\beta}(t) & =\mathrm{E}_{\beta}\left[e^{i t\left(P_{j}^{\mathrm{SU}}-b \Xi^{\mathrm{SU}}-b \Xi_{\text {out }}^{\mathrm{PU}}-b N_{0}-b P_{j}^{\mathrm{SU}}\right)}\right] \\
& =\mathrm{E}_{P_{j}^{\mathrm{SU}}}\left[e^{i t P_{j}^{\mathrm{SU}}}\right] \mathrm{E}_{\Xi} \mathrm{SU}\left[e^{-i t b \Xi^{\mathrm{SU}}}\right] \mathrm{E}_{\Xi_{\text {out }}^{\mathrm{PU}}}\left[e^{-i t b \Xi_{\text {out }}^{\mathrm{PU}}}\right] \mathrm{E}_{N_{0}}\left[e^{-i t b N_{0}}\right] \mathrm{E}_{P_{j}^{\mathrm{SU}}}\left[e^{-i t b P_{j}^{\mathrm{SU}}}\right]
\end{aligned}
$$


Considering the characteristic function of $P_{j}^{\mathrm{SU}}, \Xi^{\mathrm{SU}}, \Xi_{\text {out }}^{\mathrm{PU}}$ and $N_{0}$ the $\mathrm{CF}$ of the RV $\beta$ is written as follows

$$
\begin{aligned}
\varphi_{\beta}(t) & =\varphi_{P_{j}^{\mathrm{SU}}}(t) \cdot \varphi_{\Xi \mathrm{\Xi U}}(-b t) \cdot \varphi_{\Xi_{\text {out }}^{\mathrm{PU}}}(-b t) \cdot \varphi_{N_{0}}(-b t) \cdot \varphi_{P_{j}^{\mathrm{SU}}}(-b t) \\
& =\varphi_{P_{j}^{\mathrm{SU}}}(t) \cdot \varphi_{P_{k}^{\mathrm{SU}}}(-b t)^{\left(n^{\mathrm{SU}}-1\right)} \cdot \varphi_{\Xi_{\text {out }}^{\mathrm{PU}}}(-b t) \cdot \varphi_{N_{0}}(-b t)
\end{aligned}
$$

The distribution $f_{\beta}(x)$, can be obtained by the inverse $\mathrm{CF}$, as follows

$$
f_{\beta}(x)=\frac{1}{2 \pi} \int_{-\infty}^{\infty} \mathrm{e}^{-i x t} \varphi_{\beta}(t) \mathrm{d} t .
$$

By using (28), (31) and (32), we obtain

$$
\mathrm{P}_{S}=1-\int_{-\infty}^{0} \frac{1}{2 \pi} \int_{-\infty}^{\infty} \mathrm{e}^{-i x t} \varphi_{P_{j}}(t) \varphi_{P_{k}^{\mathrm{SU}}}(-b t)^{\left(n^{\mathrm{SU}}-1\right)} \varphi_{\Xi_{\text {out }}^{\mathrm{PU}}}(-b t) \varphi_{N_{0}}(-b t) \mathrm{d} t \mathrm{~d} x .
$$

Finally, the average number of received packets $\left(E_{\mathrm{r}}\right)$ is approximated by

$$
E_{\mathrm{r}} \approx n^{\mathrm{SU}} \cdot \mathrm{P}_{S} .
$$

Note that if the node $\mathrm{SU}_{\mathrm{rx}}$ does not perform spectrum sensing the total aggregate power should take into account the aggregate power received from the multiple transmissions from PUs located within the sensing region. Therefore, the probability of successful reception is rewritten as follows

$$
\mathrm{P}_{S}^{\prime}=\mathrm{P}\left[\frac{P_{j}^{\mathrm{SU}}}{\Xi_{i n}^{\mathrm{PU}}+\Lambda^{\mathrm{SU}}-P_{j}^{\mathrm{SU}}}>b\right] .
$$

Considering the same formulation we have adopted to derive (33), the probability of successful reception $\left(\mathrm{P}_{S}^{\prime}\right)$ when the receiver does not perform spectrum sensing is given by

$$
\mathrm{P}_{S}^{\prime}=1-\int_{-\infty}^{0} \frac{1}{2 \pi} \int_{-\infty}^{\infty} \mathrm{e}^{-i x t} \varphi_{P_{j}}(t) \varphi_{P_{k}^{\mathrm{SU}}}(-b t)^{\left(n^{\mathrm{SU}}-1\right)} \varphi_{\Xi_{\text {in }}^{\mathrm{PU}}}(-b t) \varphi_{\Xi_{\text {out }}^{\mathrm{PU}}}(-b t) \varphi_{N_{0}}(-b t) \mathrm{d} t \mathrm{~d} x .
$$

\subsection{Conditional Throughput}

The performance of the secondary network with MPR capability is characterized in this subsection. In this case, the definition of the average number of nodes successfully transmitting packets during the transmission period depends on the MPR-based PHY layer performance and on the EBS performance, as EBS influences the access of the SUs during the transmission stage.

The throughput achieved by the $\mathrm{SU}_{\mathrm{rx}}$ located in the center of the sensing region (as illustrated in Figure 1) is represented by the effective usage of the channel during the transmission period $T_{D}$ when the energy detector correctly identifies a transmission opportunity with probability $1-P_{F A}$ and with an average number of received packets of $E_{\mathrm{r}}$ given $n^{\mathrm{SU}}$ transmissions. Therefore, the utilization of the channel by the SUs' network lasts on average $T_{D}\left(1-P_{F A}\right)$ when no activity of PUs is sensed within the sensing region. A SU senses the channel during a $T_{S}$ period followed by a transmission period $T_{D}$. Hence, the conditional throughput achieved by a SU, given that no PU is active within the sensing region, is defined as the ratio between the expected utilization of the channel during the transmitting period $\left(T_{D} E_{\mathrm{r}}\left(1-P_{F A}\right)\right)$ and the frame's duration $\left(T_{S}+T_{D}\right)$. Thus, the conditional throughput is defined as follows

$$
S^{\mathrm{SU}}=\frac{T_{D} E_{\mathrm{r}}\left(1-P_{F A}\right)}{T_{S}+T_{D}} .
$$

If the node $\mathrm{SU}_{\mathrm{rx}}$ does not perform spectrum sensing, the $\mathrm{SUs}$ are allowed to transmit simultaneously during the total $T_{F}$ period, even when the PUs located within the sensing region 
transmit. Therefore, the throughput of the SUs network is equal to the average number of received packets and it is rewritten as

$$
S^{\mathrm{SU}}=E_{\mathrm{r}}^{\prime} \approx n^{\mathrm{SU}} \mathrm{P}_{S^{\prime}}^{\prime}
$$

where $\mathrm{P}_{S}^{\prime}$ is the probability of successful reception derived in (36).

\section{Performance Results and Discussion}

This section describes a set of simulations and numerical results to validate the characterization of the EBS technique performed by the SU receiver. To parameterize the energy threshold, $\gamma$, and the number of samples, $N_{S}$, the criterion presented in (25) is used, in which the $\gamma$ and $N_{S}$ are function of the probability of detection $P_{D}$. The impact of the receiver spectrum sensing and PHY layer performance on SU's wireless network throughput is studied in this section.

\subsection{Validation of the EBS Performance}

In this subsection, we assess the accuracy of the energy detector performance characterization through the comparison of the theoretical results obtained with (22) and (23) with simulation results. As described in Section 2, we consider that SUs are equipped with an energy detector. In the simulation we consider the case when PUs may randomly arrive or depart during the entire sensing period $\left(N_{S}\right)$. The PUs change their state ON/OFF according to a uniform distribution. Figure 2 depicts the simulation and theoretical results of the false alarm and detection probabilities for different values of PU's transmission power $\left(P_{T}^{\mathrm{PU}}=20 \mathrm{~dB}\right.$ and $\left.P_{T}^{\mathrm{PU}}=30 \mathrm{~dB}\right)$ and considering path loss (i.e., $\left.\alpha=2\right)$ and fading (i.e., $\sigma_{\xi}=0.7$ ) channel effects.

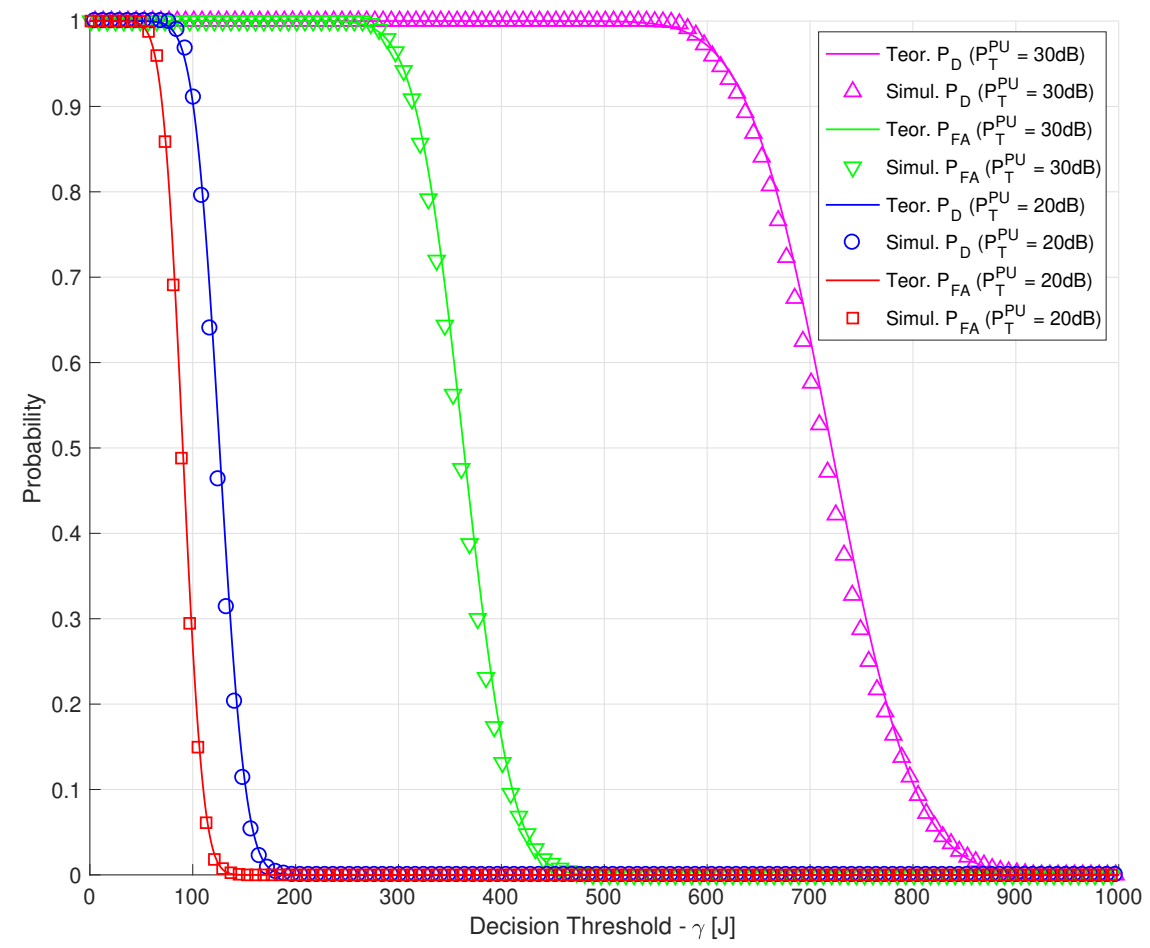

Figure 2. $P_{D}, P_{F A}$ for different thresholds $\gamma$ and $P_{T}^{\mathrm{PU}}\left(\rho_{O N}=0.5, \tau^{\mathrm{PU}}=0.001\right.$ node $/ \mathrm{m}^{2}$, $N_{S}=60$ samples, $\left.R_{G}=100 \mathrm{~m}, R_{E}=500 \mathrm{~m}, \alpha=2, \sigma_{\xi}=0.7\right)$.

From the results in Figure 2, we observe that the numerical results are close to the values obtained through simulation, which successfully validates the proposed characterization of the probabilities of detection and false alarm. For $P_{T}^{\mathrm{PU}}=20 \mathrm{~dB}$, we observe that due to the lower transmission power adopted by the PUs, $P_{D}$ and $P_{F A}$ curves are close to each other, with that the EBS technique can not operate near the optimal point of operation, where $P_{D} \approx 1$ and $P_{F A} \approx 0$. On the other hand, 
for $P_{T}^{\mathrm{PU}}=30 \mathrm{~dB}$ the descending zone of $P_{F A}$ and $P_{D}$ are more distant, meaning that the optimal operating region was extended when compared to the case when $P_{T}^{\mathrm{PU}}=20 \mathrm{~dB}$.

\subsection{Decentralized Wireless Network Performance in Shared Channels}

This subsection presents results of the performance of SU's network under different channel sensing conditions (i.e., for different path loss coefficients and levels of fading uncertainty). The results include the average number of received packets $\left(E_{\mathrm{r}}\right)$ given $n^{\mathrm{SU}}$ transmitters, and the conditional throughput of the secondary network.

We considered the MPR scenario where the $\mathrm{SU}_{\mathrm{rx}}$ can receive multiple packets simultaneously whenever the outcome of the spectrum sensing indicates the channel as being idle. We have considered the scenario illustrated in Figure 1, a SU receiver circled by SUs and PUs. Regarding the secondary network, we considered $n^{\mathrm{SU}}$ SUs transmitters located in the area $A_{i n}=\pi R_{G}^{2}$, which were distributed according to the PDF in (2). The nodes from the primary network were distributed according to a $2 \mathrm{D}$ Poisson point process. Assuming that the $\mathrm{SU}_{\mathrm{rx}}$ senses the channel as vacant, it will receive $n^{\mathrm{SU}}$ transmissions plus the total number of transmissions from the PUs located outside the sensing region (i.e., $A_{\text {out }}=\pi\left(R_{E}{ }^{2}-R_{G}{ }^{2}\right)$ ). Different noise $\left(N_{0}\right)$ and fading $\left(\Psi_{k}\right)$ realizations were used on each trial, being the receiving condition (26) observed for each SU transmitter $j$. The expected number of received packets, $E_{\mathrm{r}}$ was computed from the simulations' data. The simulations were parameterized according to the data presented in Table 1. Regarding the computation of $\mathrm{P}_{S}$ in (32) we have adopted the FFT algorithm with domain $x$ set to $[-500,500]$ and a step of $3 \times 10^{-4}$.

Table 1. Parameters used for performance evaluation of the SUs and PUs Wireless Networks.

\begin{tabular}{ll}
\hline Parameter & Value \\
\hline Channel bandwidth $(W)$ & $100 \mathrm{kHz}$ \\
Sampling Rate & $5 \mu \mathrm{s}$ \\
Frame duration $\left(T_{F}\right)$ & $10 \mathrm{~ms}$ \\
Transmission Power $\left(P_{T}^{\mathrm{SU}}\right.$ and $\left.P_{T}^{\mathrm{PU}}\right)$ & $30 \mathrm{~dB}$ \\
PUs access probability $\left(\rho_{\mathrm{ON}}\right)$ & 0.5 \\
PUs spatial density $\left(\tau^{\mathrm{PU}}\right)$ & 0.001 node $/ \mathrm{m}^{2}$ \\
$\mathrm{SU}_{\mathrm{rx}}$ sense radius $R_{G}$ & $30 \mathrm{~m}$ \\
PUs outer radius $R_{E}$ & $500 \mathrm{~m}$ \\
AWGN variance $\left(\sigma_{N_{0}}\right)$ & $1(0 \mathrm{~dB})$ \\
Capture threshold $(b)$ & 0.04 \\
\hline
\end{tabular}

Figure 3 illustrates the average number of received packets given $n^{\mathrm{SU}}$ transmitters, for different values of path loss coefficients and two levels of fading uncertainty (i.e., with and without fading). The curves identified as "Simul." represent the data obtained through simulation, while the ones identified as "Teor." were obtained by numerically computing (34). The curves identified as "Teor. without sensing" were obtained by numerically computing (38) and represents the scenario where the $\mathrm{SU}_{\mathrm{rx}}$ does not perform spectrum sensing, and as consequence, the $\mathrm{SU}$ are allowed to transmit, even when the PUs are located within the sensing region transmit (i.e., $A_{\text {in }}=\pi R_{G}^{2}$ ). In the latter scenario, the receiving condition (26) takes into account transmissions from the PUs located outside and inside of the sensing region. The SPR case is highlighted in Figure 3 with the rectangle "SPR: $n^{\mathrm{SU}}=1$ ".

From Figure 3 we observe that the numerical values of $E_{\mathrm{r}}$ closely follow the results obtained by simulation. The figure shows the maximum point of operation of the MPR-based PHY layer. After that point $E_{\mathrm{r}}$ decreases as $\alpha$ increases. The decrease is because with the increase of $\alpha$ the power propagation losses increase with the distance, meaning that the SUs further away from the $\mathrm{SU}_{\mathrm{rx}}$ receiver will experience a lower probability of successful transmission. Finally, by comparing the results of the average number of received packets for the cases with and without the spectrum sensing, we observe that the average number of received packets increases when spectrum sensing is adopted. By using 
spectrum sensing the $S U_{r x}$ achieves a better performance regarding the MPR communication, because the interference caused by the PUs' transmissions located in the sensing region is avoided.

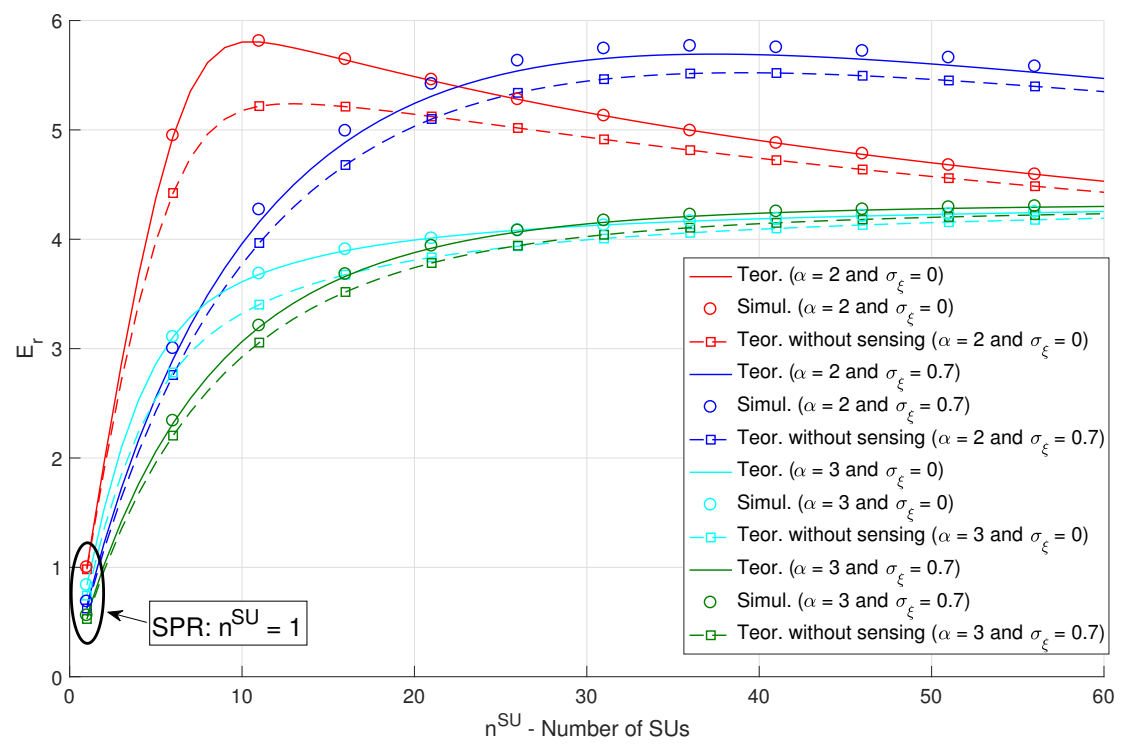

Figure 3. Average number of received packets $\left(E_{\mathrm{r}}\right)$ given $n^{\mathrm{SU}}$ transmitters, different values of $\alpha$, and with or without fading. The SPR case is highlighted in the figure with the rectangle "SPR : $n^{S U}=1$ ".

Figures 4 and 5 represent the conditional throughput by computing (37) against different values of $n^{\mathrm{SU}}$ and different parameterization of $P_{D}$. In both figures we consider two scenarios of propagation effects: path loss $(\alpha=2)$ without fading; and path loss $(\alpha=2)$ with fading $\left(\sigma_{\xi}=0.7\right)$. The $S^{\mathrm{SU}}$ without sensing was computed according to (38). Figure 4 represents the conditional throughput, $S^{\mathrm{SU}}$, for different values of $P_{D}$ and considering $n^{\mathrm{SU}}$ equal to 1,10 and 20 nodes. Note that $n^{\mathrm{SU}}$ equal to 1 node represents an SPR scenario in which the probability of successful reception only depends on the SNR. As in Figure 3, in Figure 5 the SPR scenario is represented when $n^{\mathrm{SU}}$ is equal to 1 , which is highlighted by the rectangle "SPR: $n^{\mathrm{SU}}=1$ ".

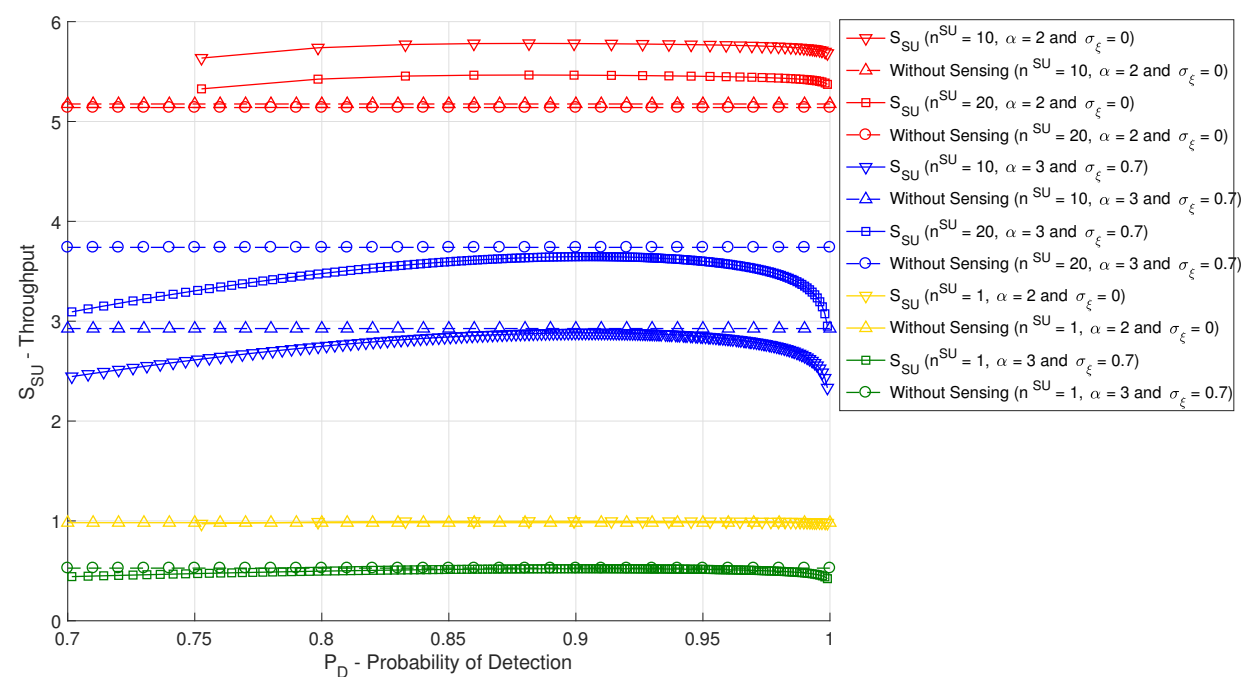

Figure 4. Conditional throughput $\left(S^{\mathrm{SU}}\right)$ achieved by SU versus $P_{D}$, for $n^{\mathrm{SU}}=\{1,10,20\}$ nodes, $\alpha=\{2,3\}$ and $\sigma_{\xi}=\{0,0.7\}$.

From Figure 4 and considering the SPR scenario (i.e., $n^{\mathrm{SU}}=1$ ), the conditional throughput of the scenarios with and without sensing are very close for both values of $\alpha$. Although results of conditional throughput do not show a huge advantage by performing a sensing technique at the receiver, it should 
be noticed that when using sensing a certain level of protection to the PUs is guaranteed. Regarding the MPR scenario, as can be seen for $\alpha$ equal to 2 and without fading the conditional throughput is higher when sensing is performed. On the other hand, for worst propagation conditions (i.e., $\alpha=3$ and $\sigma_{\xi}=0.7$ ), the conditional throughput for both cases (i.e., with and without sensing) is very similar when $P_{D}$ is between 0.85 and 0.93 . For values of $P_{D}$ lower than 0.85 the conditional throughput decrease with $P_{D}$ due to the fact that the used energy threshold criterion sets a higher probability of $P_{F A}$ as $P_{D}$ decreases. For values of $P_{D}$ close to 1 the performance of the SUs' network is degraded since the number of samples required to guarantee the level of protection to the PUs increases with $P_{D}$.

Figure 5 illustrates the conditional throughput given $n^{\mathrm{SU}}$ and for two levels of protection to the PUs' network (i.e., $P_{D}$ equal to $97.0 \%$ and $99.9 \%$ ).

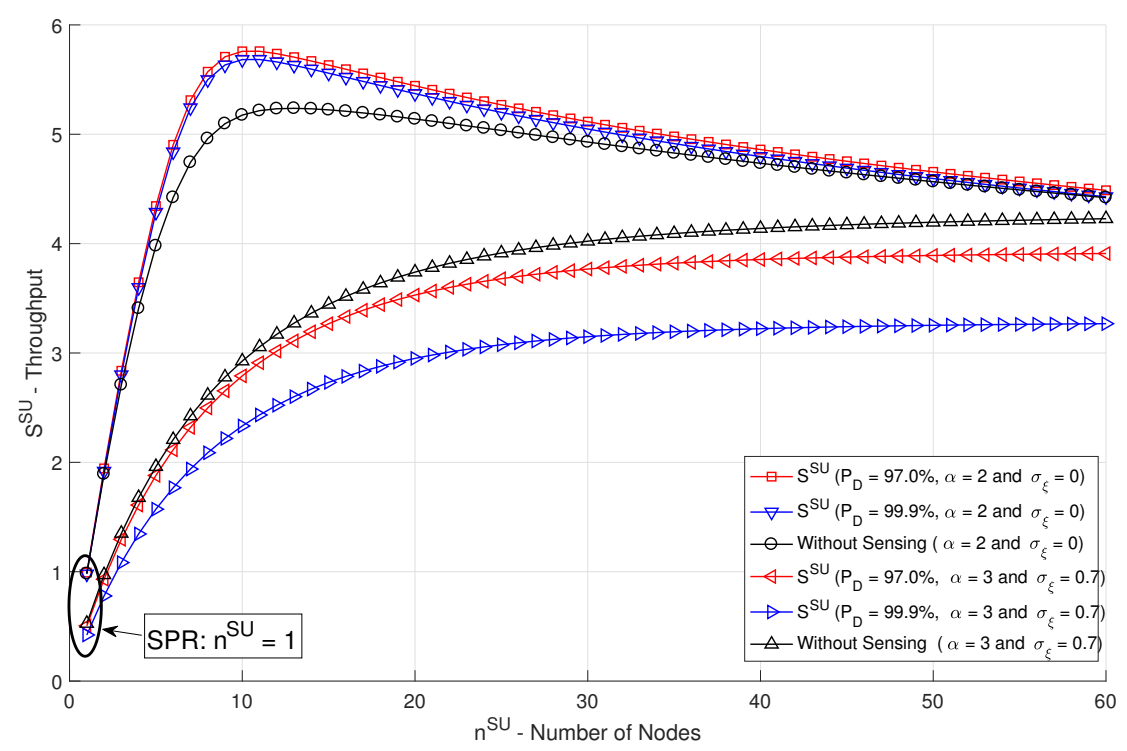

Figure 5. Conditional throughput $\left(S^{\mathrm{SU}}\right)$ achieved by $\mathrm{SU}$ versus $n^{\mathrm{SU}}$, for $P_{D}=\{97.0 \%, 99.9 \%\}$ nodes, $\alpha=\{2,3\}$ and $\sigma_{\xi}=\{0,0.7\}$. The SPR case is highlighted in the figure with the rectangle "SPR: $n^{S U}=1$ ".

From Figure 5 we observe that the channel propagation condition greatly influences $S^{\mathrm{SU}}$. Different from the results in Figure 3, where the scenario with sensing achieves higher $E_{\mathrm{r}}$ than the scenario without sensing, Figure 5 shows that $S^{\mathrm{SU}}$ in the worst propagation conditions (i.e., $\alpha=3$ and $\sigma_{\xi}=0.7$ ) decreases, since the number of samples needed to sense the channel increases.

In Figures 6 and 7 we illustrate the surface of the SUs' network conditional throughput for different values of $n^{\mathrm{SU}}$ and $P_{D}$. Figures 6 and 7 represent, respectively, the scenarios with best (i.e., $\alpha=2$ and without fading) and worst (i.e., $\alpha=3$ and $\sigma_{\xi}=0.7$ ) propagation conditions from Figure 3.

As already observed in the previous figures, from Figures 6 and 7 we observe that the conditional throughput achieved by the SUs' network is lower under more severe channel propagation conditions. The conditional throughput decreases due to two reasons: the sensing period that guarantees an optimal operation of the EBS technique (i.e., $P_{F A} \approx 0$ and $P_{D} \approx 1$ ) increases with $\alpha$; and the maximum number of successful received packets given the number of simultaneous transmitters decreases as $\alpha$ and $\sigma_{\xi}$ increase.

Based on the results of Figures 6 and 7, we conclude that the maximum SU network throughput is achieved by properly adjusting the number of simultaneous transmissions performed by the SUs and the detection probability. However, the maximum throughput does not assures full protection to the PUs network in scenarios for worst channel propagation conditions. 


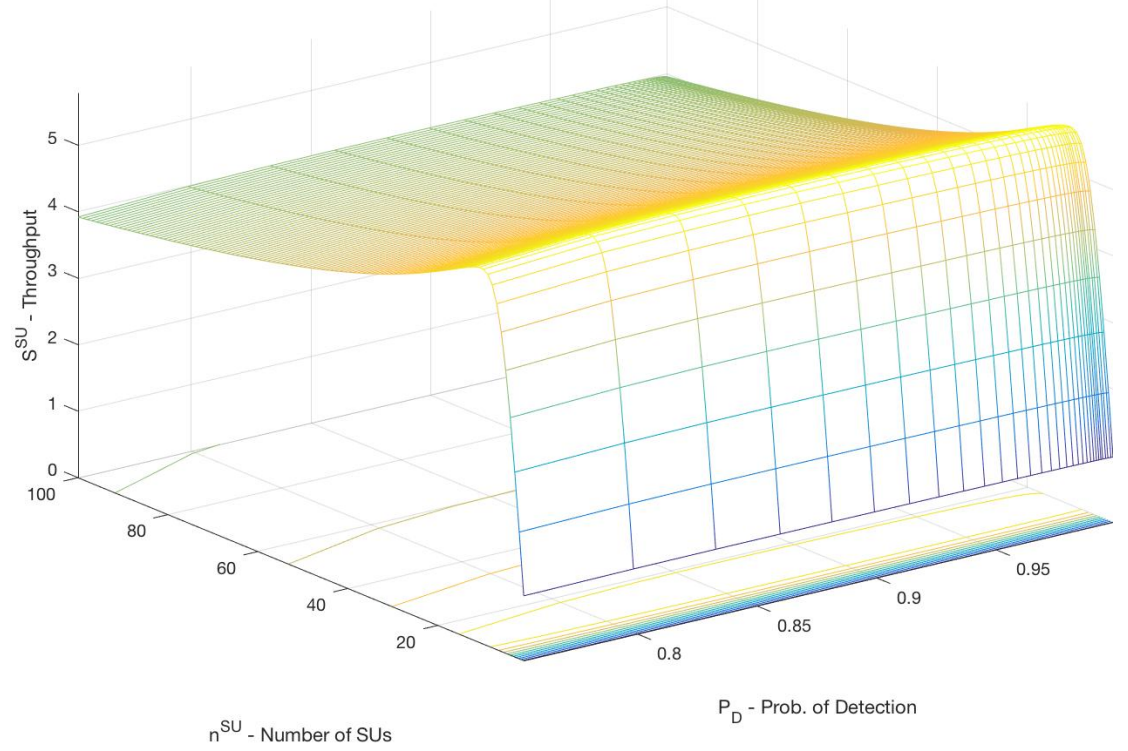

Figure 6. Conditional throughput $\left(S^{\mathrm{SU}}\right)$ achieved by $\mathrm{SU}$ versus $P_{D}$ and $n^{\mathrm{SU}}$, for $\alpha=2$ and without fading.

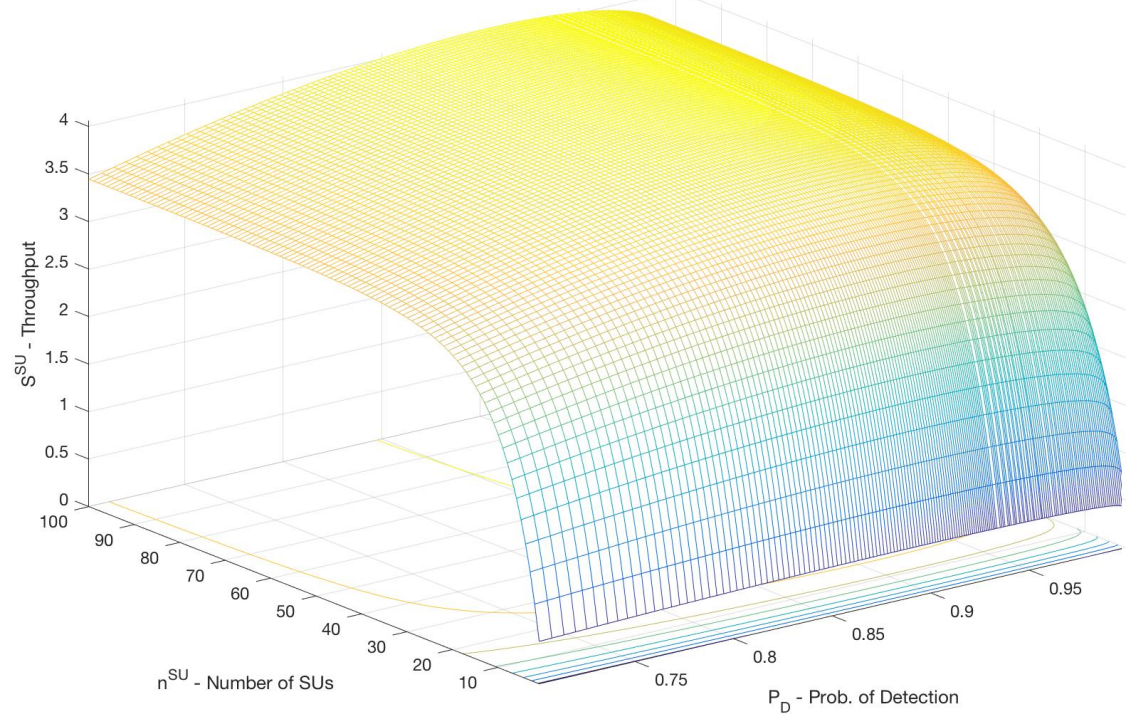

Figure 7. Conditional throughput $\left(S^{S U}\right)$ achieved by SU versus $P_{D}$ and $n^{\mathrm{SU}}$, for $\alpha=3$ and $\sigma_{\xi}=0.7$.

\section{Conclusions}

In this paper we evaluate the performance of a secondary wireless network operating in shared channels, considering that a central SU receiver performs a EBS technique to avoid interference with the surrounded primary networks and is equipped with a PHY layer with MPR capabilities. The power and amplitude of the aggregate interference caused by multiple PUs located inside and outside the sensing region were approximated by a Gamma distribution and a Normal distribution, respectively. The performance of the spectrum sensing technique was characterized through the probabilities of detection and false alarm, and closed-form expressions are presented. The results have shown that the path loss and fading propagation effects effectively impact on the SU receiver's capture capability and the secondary network performance. Both the average number of packets successfully received and the SUs conditional throughput decrease with the increase of the levels of fading uncertainty and path 
loss coefficient. This indicates that a substantial decrease of SUs network throughput is observed in worst propagation conditions. Finally, the paper also identifies the advantages of adopting spectrum sensing to avoid the interference caused by PUs that transmit within the sensing region.

Author Contributions: Conceptualization, A.F. and R.O.; software, A.F.; formal analysis, A.F. and R.O.; writing - original draft preparation, A.F and R.O.; writing-review and editing, L.B. and R.D.; funding acquisition, R.O. All authors have read and agreed to the published version of the manuscript.

Funding: This work is supported by the European Regional Development Fund (FEDER), through the Competitiveness and Internationalization Operational Programme (COMPETE 2020) of the Portugal 2020 and Programa Operacional Regional LISBOA (LISBOA 2020), and by national funds through Fundação para a Ciência e Tecnologia (FCT), under the projects CoSHARE (LISBOA-01-0145-FEDER-0307095-PTDC/EEITEL/30709/2017), InfoCent-IoT (POCI-01-0145- FEDER-030433), and project UID/EEA/50008/2019.

Conflicts of Interest: The authors declare no conflict of interest.

\section{Abbreviations}

The following abbreviations are used in this manuscript:

$\begin{array}{ll}\text { AWGN } & \text { Additive White Gaussian Noise } \\ \text { CF } & \text { Characteristic Function } \\ \text { CLT } & \text { Central Limit Theorem } \\ \text { CSMA } & \text { Carrier-Sense Multiple Access } \\ \text { EBS } & \text { Energy-based Sensing } \\ \text { IoT } & \text { Internet of Things } \\ \text { M2M } & \text { Machine-to-Machine } \\ \text { MGF } & \text { Moment-Generating Function } \\ \text { MPR } & \text { Multi-Packet Reception } \\ \text { PDF } & \text { Probability Density Function } \\ \text { PU } & \text { Primary User } \\ \text { RV } & \text { Random Variable } \\ \text { SINR } & \text { Signal-to-Interference-plus-Noise Ratio } \\ \text { SNR } & \text { Signal-to-Noise Ratio } \\ \text { SPR } & \text { Single-Packet Reception } \\ \text { SU } & \text { Secondary User }\end{array}$

\section{References}

1. Rajandekar, A.; Sikdar, B. A Survey of MAC Layer Issues and Protocols for Machine-to-Machine Communications. IEEE Internet Things J. 2015, 2, 175-186. [CrossRef]

2. Gupta, P.; Kumar, P. The capacity of wireless networks. IEEE Trans. Inf. Theory 2000, 46, 388-404. [CrossRef]

3. Zhang, Y.; Gong, A.; Lo, Y.; Li, J.; Shu, F.; Wong, W.S. Generalized $p$-Persistent CSMA for Asynchronous Multiple-Packet Reception. IEEE Trans. Commun. 2019, 67, 6966-6979. [CrossRef]

4. Zhang, Y.; Lo, Y.; Shu, F.; Li, J. Achieving Maximum Reliability in Deadline-Constrained Random Access With Multiple-Packet Reception. IEEE Trans. Veh. Technol. 2019, 68, 5997-6008. [CrossRef]

5. Sadjadpour, H.; Wang, Z.; Garcia-Luna-Aceves, J. The capacity of wireless ad hoc networks with multi-packet reception. IEEE Trans. Commun. 2010, 58, 600-610. [CrossRef]

6. MacKenzie, R.; O'Farrell, T. Throughput and Delay Analysis for p-Persistent CSMA with Heterogeneous Traffic. IEEE Trans. Commun. 2010, 58, 2881-2891. [CrossRef]

7. Dai, L. Toward a Coherent Theory of CSMA and Aloha. IEEE Trans. Wirel. Commun. 2013, 12, 3428-3444. [CrossRef]

8. Sun, X.; Dai, L. To Sense or Not To Sense: A Comparative Study of CSMA With Aloha. IEEE Trans. Commun. 2019, 67, 7587-7603. [CrossRef]

9. Zhang, Y.J.; Zheng, P.X.; Liew, S.C. How Does Multiple-Packet Reception Capability Scale the Performance of Wireless Local Area Networks? IEEE Trans. Mob. Comput. 2009, 8, 923-935. [CrossRef]

10. Jin, H.; Seo, J.; Leung, V.C.M. Cooperative Pseudo-Bayesian Backoff Algorithms for Unsaturated CSMA Systems with Multi-Packet Reception. IEEE Trans. Mob. Comput. 2015, 14, 302-315. [CrossRef] 
11. Wang, H.; Fapojuwo, A.O. A Survey of Enabling Technologies of Low Power and Long Range Machine-to-Machine Communications. IEEE Commun. Surv. Tutor. 2017, 19, 2621-2639. [CrossRef]

12. Bae, Y.H.; Choi, B.D.; Alfa, A.S. Achieving Maximum Throughput in Random Access Protocols with Multipacket Reception. IEEE Trans. Mob. Comput. 2014, 13, 497-511. [CrossRef]

13. Chan, D.S.; T. Berger, T.; Tong. L. Carrier Sense Multiple Access Communications on Multipacket Reception Channels: Theory and Applications to IEEE 802.11 Wireless Networks. IEEE Trans. Commun. 2013, 61, 266-278. [CrossRef]

14. Chen, C.; Hou, S.; Wu, S. A Novel Analytical Model for Asynchronous Multi-Packet Reception MAC Protocol. IEEE Commun. Lett. 2017, 21, 1289-1292. [CrossRef]

15. Stefanović, C.; Paolini, E.; Liva, G. Asymptotic Performance of Coded Slotted ALOHA With Multipacket Reception. IEEE Commun. Lett. 2018, 22, 105-108. [CrossRef]

16. Choi, J. On Simultaneous Multipacket Channel Estimation and Reception in Random Access for MTC Under Frequency-Selective Fading. IEEE Trans. Commun. 2018, 66, 5360-5369. [CrossRef]

17. Zhao, L.; Chi, X.; Qian, L.; Chen, W. Analysis on Latency-Bounded Reliability for Adaptive Grant-Free Access With Multipackets Reception (MPR) in URLLCs. IEEE Commun. Lett. 2019, 23, 892-895. [CrossRef]

18. Furtado, A.; Irio, L.; Oliveira, R.; Bernardo, L.; Dinis, R. Spectrum Sensing Performance in Cognitive Radio Networks With Multiple Primary Users. IEEE Trans. Veh. Technol. 2016, 65, 1564-1574. [CrossRef]

19. Stüber, G L. Principles of Mobile Communication, 2nd, ed.; Springer: Boston, MA, USA, 2001.

20. Al-Ahmadi, S.; Yanikomeroglu, H. On the approximation of the generalized-K distribution by a gamma distribution for modeling composite fading channels. IEEE Trans. Wirel. Commun. 2010, 9, 706-713. [CrossRef]

21. Irio, L.; Furtado, A.; Oliveira, R.; Bernardo, L.; Dinis, R. Interference Characterization in Random Waypoint Mobile Networks. IEEE Trans. Wirel. Commun. 2018, 17, 7340-7351. [CrossRef]

22. Tang, H. Some physical layer issues of wide-band cognitive radio systems. In Proceedings of the First IEEE International Symposium on New Frontiers in Dynamic Spectrum Access Networks, 2005, DySPAN 2005, Baltimore, MD, USA, 8-11 November 2005; pp. 151-159.

23. Ghasemi, A.; Sousa, E. Optimization of Spectrum Sensing for Opportunistic Spectrum Access in Cognitive Radio Networks. In Proceedings of the 2007 th IEEE Consumer Communications and Networking Conference, Las Vegas, NV, USA, 11-13 January 2007; pp. 1022-1026. [CrossRef]

24. Luis, M.; Furtado, A.; Oliveira, R.; Dinis, R.; Bernardo, L. Towards a Realistic Primary Users' Behavior in Single Transceiver Cognitive Networks. IEEE Commun. Lett. 2013, 17, 309-312. [CrossRef]

25. Kostylev, V. Energy detection of a signal with random amplitude. In Proceedings of the IEEE International Conference on Communications, ICC 2002, New York, NY, USA, 28 April-2 May 2002; Volume 3, pp. 1606-1610. [CrossRef]

26. Furtado, A.; Oliveira, R.; Dinis, R.; Bernardo, L. Successful Packet Reception Analysis in Multi-Packet Reception Wireless Systems. IEEE Commun. Lett. 2016, 20, 2498-2501. [CrossRef] 